\title{
Review
}

\section{The Spanish biology/disease initiative within the human proteome project: Application to rheumatic diseases ts}

\author{
Cristina Ruiz-Romero ${ }^{a, b}$, Valentina Calamia ${ }^{a}$, Juan Pablo Albar, ${ }^{c}+$, José Ignacio Casal ${ }^{d}$, \\ Fernando J. Corrales ${ }^{e}$, Patricia Fernández-Puente ${ }^{a}$, Concha Gil ${ }^{f}$, Jesús Mateos ${ }^{a}$, \\ Fernando Vivanco ${ }^{g}$, Francisco J. Blanco ${ }^{a, h, *}$
}

${ }^{a}$ Rheumatology Division, ProteoRed/ISCIII Proteomics Group, INIBIC - Hospital Universitario de A Coruña, 15006 A Coruña, Spain

${ }^{\mathrm{b}}$ CIBER-BBN Instituto de Salud Carlos III, INIBIC-CHUAC, A Coruña, Spain

'ProteoRed-ISCIII, Centro Nacional de Biotecnología - CSIC, UAM Campus Cantoblanco, Darwin, 3, 28049 Madrid, Spain

${ }^{d}$ ProteoRed-ISCIII, Functional Proteomics, Department of Cellular and Molecular Medicine, Centro de Investigaciones Biológicas (CIB-CSIC), Ramiro de Maeztu 9, 28040 Madrid, Spain

eProteoRed-ISCIII, Center for Applied Medical Research (CIMA), University of Navarra, Pío XII, 55; Ed. CIMA, 31008 Pamplona, Spain

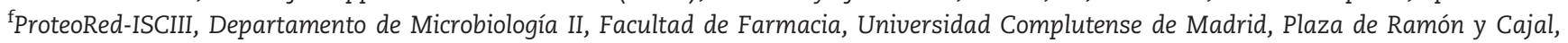
28040 Madrid, Spain

groteoRed-ISCIII, Department of Immunology, IIS-Fundacion Jimenez Diaz, Madrid, Spain

${ }^{h}$ RIER-RED de Inflamación y Enfermedades Reumáticas, INIBIC-CHUAC, A Coruña, Spain

\section{A R T I C L E I N F O}

\section{Keywords:}

Human proteome project

Rheumatic diseases

Cartilage

Chondrocyte

Synovial fluid

Osteoarthritis

\begin{abstract}
A B S T R A T
The Spanish Chromosome 16 consortium is integrated in the global initiative Human Proteome Project, which aims to develop an entire map of the proteins encoded following a gene-centric strategy (C-HPP) in order to make progress in the understanding of human biology in health and disease (B/D-HPP). Chromosome 16 contains many genes encoding proteins involved in the development of a broad range of diseases, which have a significant impact on the health care system. The Spanish HPP consortium has developed a B/D platform with five programs focused on selected medical areas: cancer, obesity, cardiovascular, infectious and rheumatic diseases. Each of these areas has a clinical leader associated to a proteomic investigator with the responsibility to get a comprehensive understanding of the proteins encoded by Chromosome 16 genes. Proteomics strategies have enabled great advances in the area of rheumatic diseases, particularly in osteoarthritis, with studies performed on joint cells, tissues and fluids.
\end{abstract}

\footnotetext{
is This article is part of a Special Issue entitled: HUPO 2014.

* Corresponding author at: INIBIC-Complejo Hospitalario Universitario A Coruña, C/Xubias, 84, 15006-A Coruña, Spain. Tel.: + 34 981 176399; fax: +34 981176398.

E-mail address: fblagar@sergas.es (F.J. Blanco).

† In memoriam of Juan Pablo Albar.
} 
Biological significance

In this manuscript we describe how the Spanish HPP-16 consortium has developed a B/D platform with five programs focused on selected medical areas: cancer, obesity, cardiovascular, infectious and rheumatic diseases. Each of these areas has a clinical leader associated to a proteomic investigator with the responsibility to get a comprehensive understanding of the proteins encoded by Chromosome 16 genes. We show how the Proteomic strategy has enabled great advances in the area of rheumatic diseases, particularly in osteoarthritis, with studies performed on joint cells, tissues and fluids.

This article is part of a Special Issue entitled: HUPO 2014.

(c) 2015 Elsevier B.V. All rights reserved.

\section{Contents}

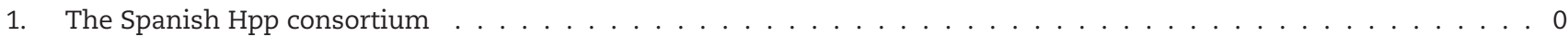

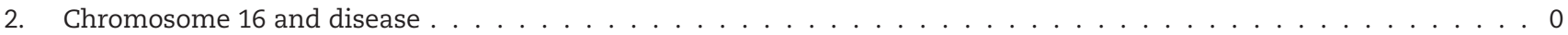

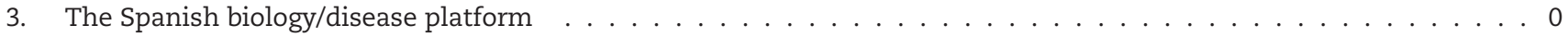

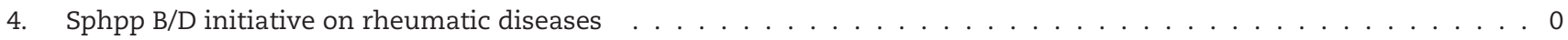

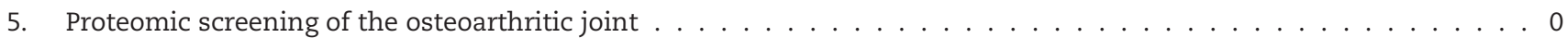

6. Development of the Hpp pillars in osteoarthritis research . . . . . . . . . . . . . . . . . . . . . . . . . . . 0

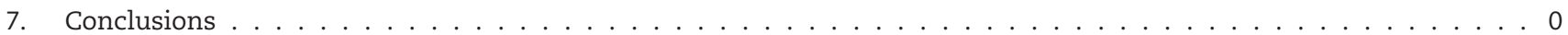

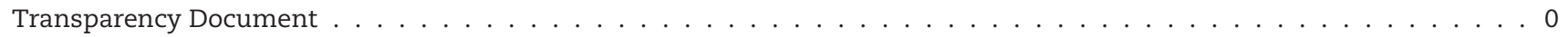

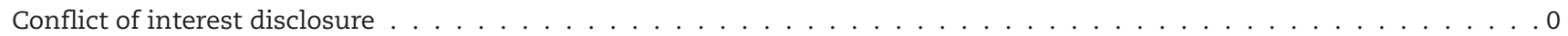

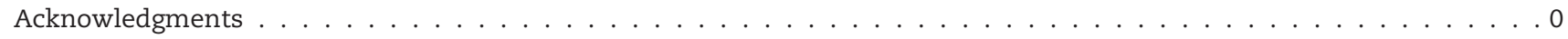

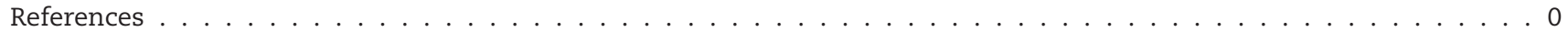

\section{The Spanish Hpp consortium}

Since its foundation, the Spanish Human Proteome Project consortium (SpHPP) is structured on two platforms on the basis of ProteoRed-ISCIII network (Fig. 1). This consortium is integrated in the global initiative Human Proteome Project [1], which aims to develop an entire map of the proteins encoded following a genecentric strategy (C-HPP) [2] in order to make progress in the understanding of human biology in health and disease (B/D-HPP).

The SpHPP project was conceived on a multi-centric configuration, assuming the standards and integration procedures already available in ProteoRed-ISCIII (http://www.proteored. org), which are encompassed with the HUPO initiatives. Within this consortium, the Spanish C-HPP platform aims to identify and characterize previously known and unknown proteins of Chromosome 16, whereas the B/D-HPP platform aims the cooperation of basic researchers with clinical groups belonging to networks of the Spanish Institute of Health (ISCIII): CIBERs (Networks of Biomedical Research Centers) and RETICS (Thematic Networks of Cooperative Health Research). This will facilitate not only the comprehensive study of the products of the 836 protein-coding genes in Chromosome 16 in diverse sample types (cells, tissues or biofluids), but also their possible role in disease pathogenesis, as well as their putative usefulness for the development of diagnosis, prognosis and treatment strategies.

\section{Chromosome 16 and disease}

Alterations in Chromosome 16, which comprises near 900 proteins, have been linked to several different disorders such as obesity, autism, neurodegenerative diseases, cancer, thalassemia or spondyloarthropaties. Genetics Home Reference (http://ghr.nlm.nih.gov) lists 58 conditions that are related to genes on Chromosome 16. These conditions include not only common hemoglobinopathies such as alpha-thalassemia, but also different types of cancers, inflammatory bowel diseases such as Crohn and a number of metabolism-related syndromes. A comprehensive proteomic analysis of Chr. 16 proteins will be highly valuable for a better understanding of these pathologies.

\section{The Spanish biology/disease platform}

With the aim of coordinating all those efforts related with biology and disease driven projects within the HPP in Spain, the SpHPP consortium has developed a BD platform with a manager and five programs focused in selected medical areas, based on those genes located in the Chromosome 16 (Fig. 2): cancer, obesity, cardiovascular, infectious and rheumatic diseases. Each of these areas has a clinical leader (oncologist, endocrinologist, cardiologist, internal medical specialist and rheumatologist), and are co-chaired by a ProteoRed-ISCIII member.

Clinicians are associated to proteomic investigators with the responsibility to get a comprehensive understanding of the proteins encoded by the genes and their relationship with disease. Based on the genes located in the Chromosome 16 , ten diseases (colon cancer, brain cancer, breast cancer, obesity, atherosclerosis, candidiasis, rheumatoid arthritis and osteoarthritis) have been selected to acquire significant medical benefits, including improved capabilities for early detection and diagnosis, therapeutic development and monitoring and personalized healthcare programs. 


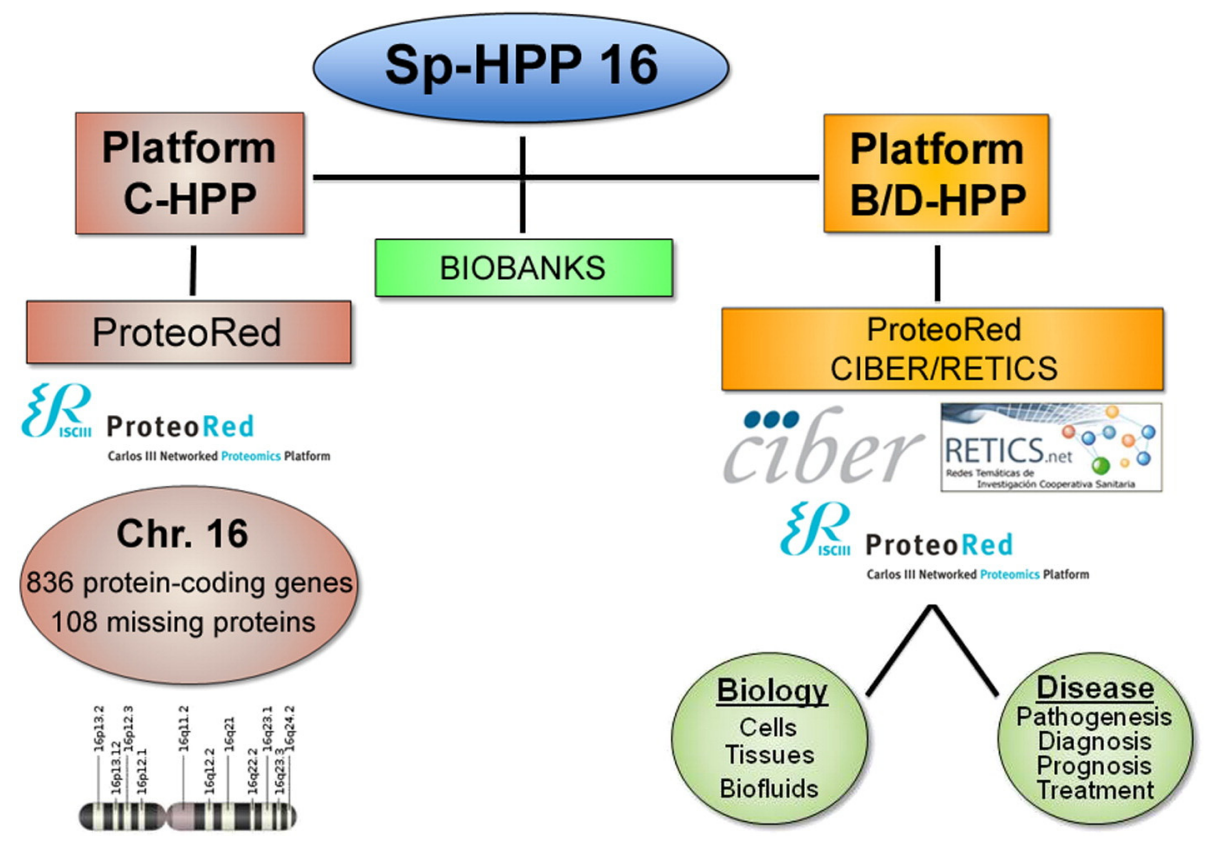

Fig. 1 - The SHPP-16 consortium. The C-HPP is structured on the basis of ProteoRed network, and its main objective is the identification and proteomic characterization of previously known and unknown proteins encoded on Chromosome 16. The B/D-HPP platform aims the cooperation of ProteoRed researchers with clinical groups belonging to networks of the Spanish Institute of Health (ISCIII): CIBER and RETICS. This will facilitate not only the comprehensive study of proteins encoded on the Chromosome 16 in diverse sample types (cells, tissues or biofluids), but also their possible role in disease pathogenesis, or their putative usefulness for the development of diagnosis, prognosis and treatment strategies.

The Clinical Research that is essential to validate the proposed biomarkers on each medical area will be carried out using the national clinical networks of the Spanish Health Administration (National Institute of Health, ISCIII) such as the CIBER on obesity (CIBEROBN) and different RETICS: Cardiovascular Network (RIC), Cancer National Network (RTICC), Infectious Pathologies Network (REIPI) and National Network of Inflammation and Rheumatic Diseases (RIER).

Each medical area will focus on one to three specific diseases (Fig. 2). As the quality of biological samples is a critical point to obtain high confidence results in the HPP project, the Spanish National Biobank Network (also belonging to the Carlos III National Health Institute) has been involved in the collection and storage of all selected biological fluids and tissues that are needed to carry out clinical research in the B/D-HPP platform, and will be the provider of clinical samples to the project.

\section{Sphpp B/D initiative on rheumatic diseases}

Among the five medical areas of the Spanish B/D initiative, this article will then focus on describing the work performed in the area of rheumatic diseases. Rheumatism is a non-specific term used to describe any painful disorder affecting the loco-motor system including joints, muscles, connective tissues and soft tissues around the joints and bones. Rheumatologic disorders include highly prevalent pathologies such as osteoarthritis, inflammatory diseases such as rheumatoid arthritis, psoriatic arthritis or spondyloarthropaties, and autoimmune diseases such as systemic lupus erythematosus (SLE), Behçet 's syndrome or sarcoidosis.

There are several genes encoded in Chromosome 16 with a possible reported role in the pathogenesis of a number of rheumatologic disorders, which can be classified into three groups of diseases (Table 1): A, age-related rheumatic pathologies; $\mathrm{B}$, inflammatory arthritis; and $\mathrm{C}$, autoimmune disorders.

Osteoarthritis (OA, Group A), is the most frequent arthropathy, ultimately affecting more than $10 \%$ of the population and being the leading cause of permanent work incapacity. It is characterized by the progressive degradation of hyaline articular cartilage and is associated with aging [3]. Among inflammatory arthritis (Group B), Rheumatoid arthritis (RA) is a chronic systemic inflammatory disease of undetermined etiology involving primarily the synovial membranes and articular structures of multiple joints. It is associated with progressive and severe disability and early death, mainly due to not only increased cardiovascular disease but also to many other comorbidities. A number of proteomic studies have been performed to elucidate the pathogenic mechanisms of this disease, and also to search for novel biomarkers useful for therapy monitoring [4,5]. Apart from RA, other relevant arthritis such as psoriasic arthritis (PA) or spondyloarthropaties (SpAs) should be taken into account. In this sense, a direct link between mutations in the NOD2 gene (located on chr. 16) and Crohn's disease has been recently found [6]. Interestingly, this disease has a probable autoimmune origin and is associated with the development of SpAs. Finally, other characteristic autoimmune disorders will be the subject of our studies (Group C, Table 1). Among these, SLE is an autoimmune connective 


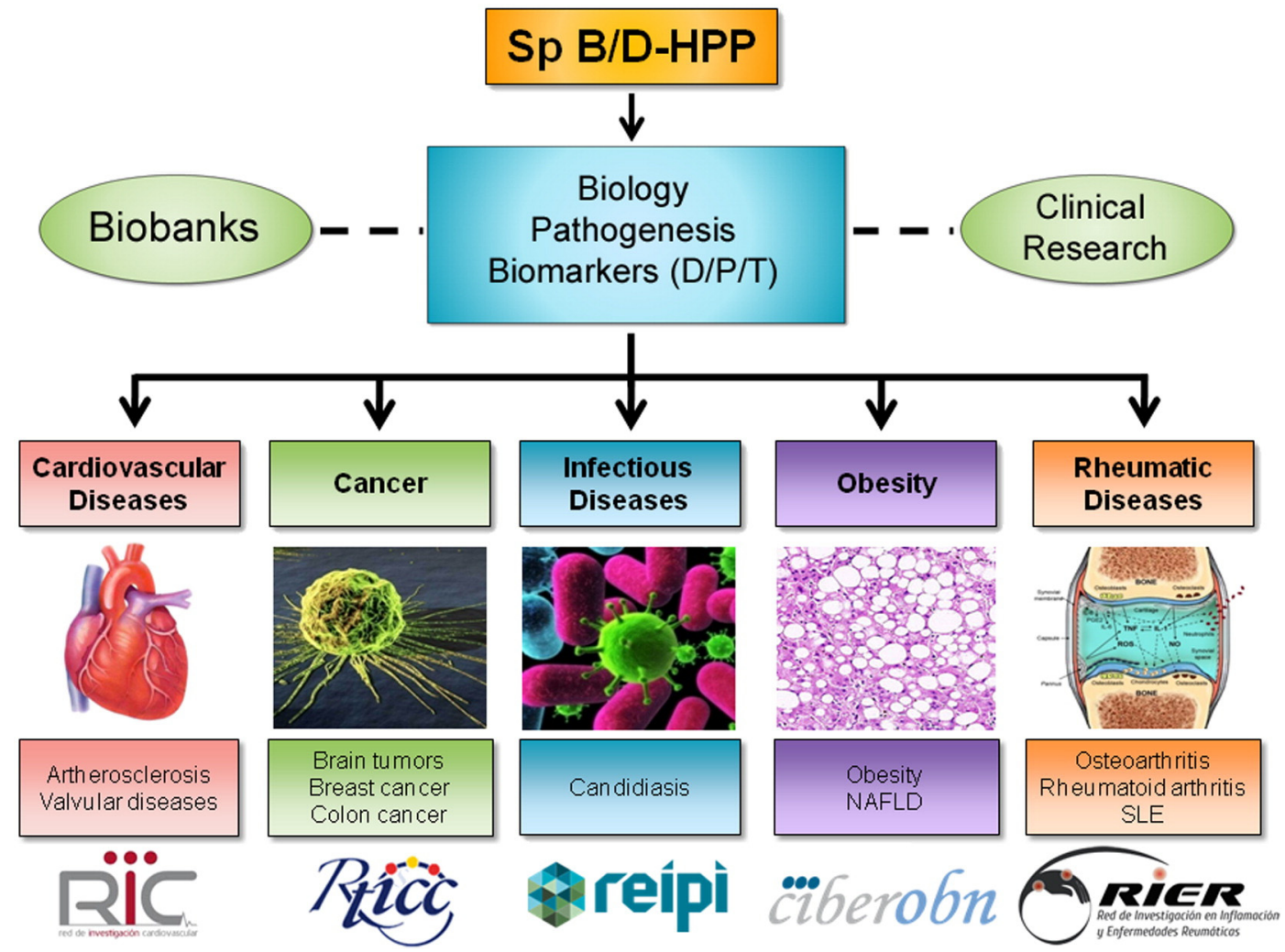

Fig. 2 - The Spanish B/D-HPP platform. Five medical areas have been selected, based on the genes located in the Chromosome 16. These are led by a clinician and co-chaired by a ProteoRed member. Each medical area will focus on one to three specific diseases (NAFLD: non-alcoholic fatty liver disease; SLE: systemic lupus erythematosus). Clinical samples needed to perform the experiments will be stored and provided by the Spanish National Biobank Network. Clinical research to validate the proposed disease biomarkers in each medical area will be carried out using the national clinical networks of the Spanish Health Administration (Carlos III National Institute of Health, ISCIII): CIBER (CiberOBN) and RETICS (RIC, RTICC, REIPI and RIER).

tissue disease of unknown origin affecting virtually all organ systems, with no specific cause but a number of environmental triggers and a number of genetic susceptibilities. The

Table 1-Genes encoded on Chromosome 16 that are related with rheumatic diseases.

\begin{tabular}{|c|c|}
\hline Disease & Genes in Chromosome 16 \\
\hline \multicolumn{2}{|l|}{ A. Aging } \\
\hline Osteoarthritis $(\mathrm{OA})$ & $\begin{array}{l}\text { Heparan sulfate synthases } \\
\text { (HS3ST2, } 4 \text { and 6) } \\
\text { Chondroitinsulfatase (GALNS) } \\
\text { MMPs (MMP2, } 15 \text { and 25) } \\
\text { CLUAP1 } \\
\text { Proteins related with the } \\
\text { mitochondrial } \\
\text { function }\end{array}$ \\
\hline \multicolumn{2}{|l|}{ B. Arthritis } \\
\hline Rheumatoid arthritis (RA) & ILs (IL-17, 27, 32 and 34) \\
\hline Psoriatic arthritis & ILRs (IL4R and 21R) \\
\hline Spondyloarthropaties & $\begin{array}{l}\text { TNFRs (TNFRSF12A and 17) } \\
\text { NOD2 }\end{array}$ \\
\hline \multicolumn{2}{|l|}{ C. Autoimmune disorders } \\
\hline Systemic lupus erythematosus (SLE) & CIITA \\
\hline Beçet's disease & $\begin{array}{l}\text { Integrin precursors } \\
\text { (ITGA-D, L, M and X) }\end{array}$ \\
\hline Sarcoidosis & $\begin{array}{l}\text { Complement components } \\
\text { (C1QTNF8) }\end{array}$ \\
\hline
\end{tabular}

understanding of the genetic basis of SLE has been rapidly advanced using large-scale, case-control, candidate gene studies as well as genome-wide association studies during the last years [7]. One of the best known associations was found between SLE susceptibility and ITGAM or ITGAM-ITGAX region [8], which encodes integrin alpha-M (also known as CD11b or complement receptor 3 ), the $\alpha$-chain of the aM $\beta 2$ integrin. Both ITGAM and ITGAX are localized in Chromosome 16.

\section{Proteomic screening of the osteoarthritic joint}

As a starting point for the study of rheumatic diseases from the perspective of the HPP project, our group has performed in the recent years several proteomic analyses on cells, tissues and biofluids from the osteoarthritic joint, in order to increase the knowledge about the pathogenesis of this disease [9], and also to discover novel OA biomarker candidates [10] (Fig. 3).

The first studies were carried out on human articular chondrocytes (which are the only cell type resident in mature cartilage) in primary culture. These works led to the definition of a panel of chondrocyte intracellular proteins that are quantitatively altered in osteoarthritic cells compared to healthy controls [11] (including chaperones and glycolytic proteins), and also to the characterization of the chondrocyte's 


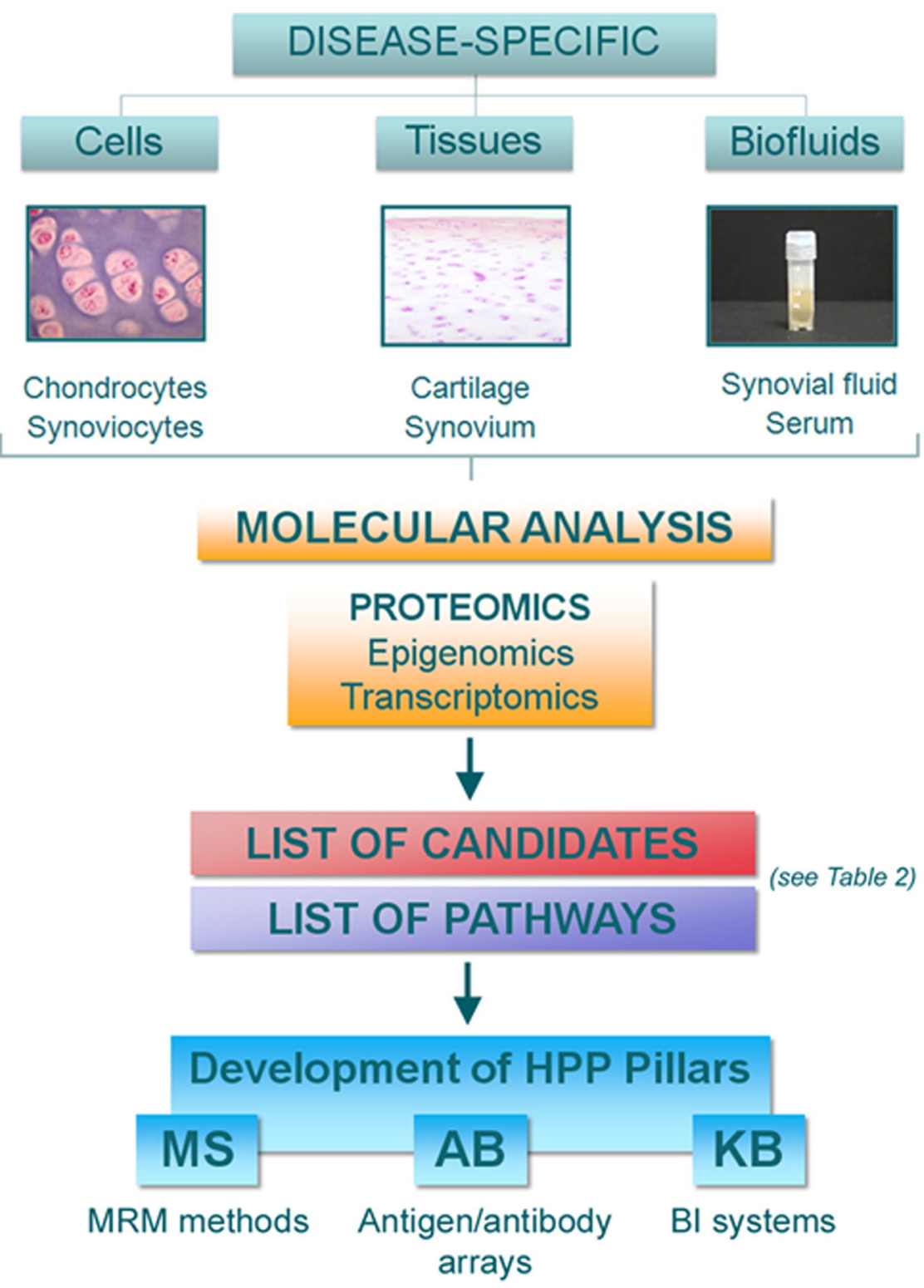

Fig. 3 - Workflow of the Spanish B/D-HPP initiative on rheumatic diseases. Proteomic screening on disease-specific cells, tissues and biofluids has led to the definition of a series of candidates related with different biological pathways (an example of ten of these candidates is shown in Table 2). The development of the three HPP pillars is currently being performed: MS, through the design of targeted MRM methods and its application on clinical cohorts; AB, through the fabrication of antigen and antibody arrays and its application on clinical cohorts; and $\mathrm{KB}$, through the creation of business intelligence (BI) systems for knowledge management.

response against hypoxic conditions [12], proinflammatory mediators such as IL-1 $\beta$ or TNF- $\alpha$ [13], or drugs intended for the treatment of osteoarthritis [14]. Apart from these studies on whole chondrocytic proteins, proteomic profiling of chondrocyte mitochondria was also carried out $[15,16]$. These latter works unraveled the mitochondrial dysregulation that occurs in osteoarthritis, which has been previously reported and was further explored in other studies [17,18]. Finally, additional assays were performed using the SILAC approach to investigate the protein profiles of the subset of chondrocyte secreted proteins (secretome) in response to IL-1 $\beta$ [19] or anti-OA drugs [20,21], considering the essential role of this cell in synthesizing the cartilage extracellular matrix and maintaining its proper turnover. All these studies provide key information about the mechanisms participating in osteoarthritis pathogenesis, and also those driven in cartilage cells by either proinflammatory or therapeutic agents.

Furthermore, proteomic analyses on human articular cartilage have also been performed. This highly specialized tissue has an organized architecture and is composed mainly (95-98\%) of a dense extracellular matrix (ECM) including collagens and proteoglycans that make the protein extraction very difficult. In spite of this, some studies have compared the protein profiles of healthy and osteoarthritic tissue [22,23]. Furthermore, other reports have focused on the peptides or proteins released to the culture medium from cartilage explants. 
Most of these works showed the effect on human tissue of metalloproteases [24] or cytokines [25] as in vitro models of disease. Very recently, our group has conducted an analogous study, but comparing the protein profiles released from cartilages of healthy donors, macroscopically intact tissue of OA patients, and damaged tissue from the same individuals [26].

The proteins depicted in the above-mentioned works may also have biomarker utility, as they are altered in chondrocytes or cartilage as a consequence of the disease or the cellular response to an outer stimulus. In this sense, great efforts are being made in the recent years for the search of novel biomarkers useful for diagnosis, prognosis and monitorization of therapeutic responses in osteoarthritis [27]. Ideally, biochemical markers are derived from body fluids where they can easily be measured and monitored, such as serum, plasma or urine, and also from joint-specific samples such as synovial fluid. Using serum cohorts from OA patients and controls, some studies have identified differential proteins with putative biomarker value by SELDI-TOF $[28,29]$ and also following an iTRAQ-based approach [30]. Alternatively, synovial fluid provides the advantage of being located where the disease occurs, thus containing higher concentrations of cartilage degradation products compared to other body fluids, but have the drawback of being more difficult to obtain (especially from healthy donors or at early disease stages). Therefore, some proteomic studies have analyzed OA synovial fluid using samples from rheumatoid arthritis patients as comparator group [31,32] and only two works have addressed differences between $\mathrm{OA}$ and healthy controls by shotgun proteomics $[33,34]$, both using a small amount of samples for the analysis.

\section{Development of the Hpp pillars in osteoarthritis research}

All those shotgun studies reported above have led to the definition of a series of proteins related with the pathogenesis of osteoarthritis, or putative biomarker candidates for early diagnosis, prognosis and development of drugs for OA treatment. As an example of this, Table 2 shows the top 10 proteins most commonly identified in our shotgun studies as altered in osteoarthritis disease, either in human articular chondrocytes [19], articular cartilage [26], synovial fluid [4] or serum [30]. Interestingly, while some of these proteins have been already proposed as OA biomarkers and are currently undergoing qualification studies (such as COMP [35] or YKL-40 [36]); most of them are novel biomarker candidates for OA. Multiplexed evaluations of these panels of proteins are needed to advance further in the OA biomarker pipeline [10]. For this task, targeted proteomics technologies are highly valuable, not only because of their capacity for simultaneously analyzing multiple analytes, but also because of their potential applicability in high-throughput studies such as clinical trials [37]. Active research will be the key for facilitating the categorization of $\mathrm{OA}$ biomarkers using the BIPEDS classification scheme that has been developed as framework for communication in the field [38,39]: burden of disease, investigative, prognostic, efficacy of intervention, diagnostic or safety markers. Therefore, attention should now be focused on the implementation of the three pillars of HPP [1] (mass spectrometry, affinity proteomics and knowledgebase) in order to develop improved methods and technologies that facilitate the analysis of these candidates. This will be essential to advance further in the understanding of the mechanisms involved in disease, and also to qualify the biomarker utility of the target proteins [40].

Regarding the MS pillar, the development of selected/ multiple reaction monitoring (SRM/MRM) in the context of the HPP [41], and its increasingly frequent application in clinical studies [42] is a highly valuable tool for alleviating the existing bottleneck in moving markers from discovery phases to the clinical arena. In fact, targeted analyses based on this promising technology have recently begun to be conducted for assessing the levels of specific proteins in observational OA cohorts [43].

Table 2-Top 10 proteins detected as altered in the proteomic analyses performed by our group for the search of osteoarthritis biomarkers.

\begin{tabular}{|c|c|c|c|c|c|}
\hline Acc. no. & Protein name & Chondrocytes [19] & Cartilage [26] & $\begin{array}{l}\text { Synovial } \\
\text { fluid [31] }\end{array}$ & Serum [30] \\
\hline \multicolumn{6}{|c|}{ GO:0001501 Skeletal system development } \\
\hline P16112 & Aggrecan core protein & Down & Down & ND & Up \\
\hline P49747 & Cartilage oligomeric matrix protein & ND & Up & Up & Up \\
\hline P05452 & Tetranectin & Down & Up & Up & Up \\
\hline \multicolumn{6}{|c|}{ GO:0051216 Cartilage development } \\
\hline P51884 & Lumican & Up & Up & ND & Up \\
\hline P36222 & Chitinase-3-like protein 1 (YKL-40) & Up & Up & ND & Up \\
\hline \multicolumn{6}{|c|}{ GO:0007155 Cell adhesion } \\
\hline P07996 & Thrombospondin-1 & Up & ND & ND & Up \\
\hline P02751 & Fibronectin & Up & Up & ND & Up \\
\hline \multicolumn{6}{|c|}{ GO:0006955 Immune response } \\
\hline Q92954 & Proteoglycan 4 & Up & Up & ND & Down \\
\hline P00746 & Complement factor D & ND & ND & Up & Up \\
\hline \multicolumn{6}{|c|}{ GO:0050728 Inflammatory response } \\
\hline P36955 & Pigment epithelium-derived factor & ND & Up & ND & Up \\
\hline
\end{tabular}


Furthermore, efforts for the improvement of multiplexed, high-throughput technologies that can bridge the gap between basic research and in vitro diagnostic methods have also been made in the area of affinity proteomics (AB pillar), especially with the development of protein arrays and other antibody-based profiling studies [44]. Different screening strategies using protein arrays have been already applied for profiling autoantibodies, cytokines, and bone-turnover products in rheumatoid arthritis [45]. In osteoarthritis, we have carried out a very recent study employing antigen microarrays and NAPPA (nucleic acid programmable protein arrays) to characterize the differential autoantibody profiles in samples from OA, rheumatoid arthritis (RA) and healthy controls [46]. At present, further studies are being performed using antibody arrays for the screening of larger cohorts in order to verify putative OA biomarker candidates (Lourido et al., unpublished results).

Finally, the bioinformatics-driven knowledgebase (KB) pillar of HPP draws upon resources that already exist such as SwissProt/Uniprot, PRIDE, Peptide Atlas or Human Protein Atlas databases. Although $\mathrm{KB}$ resources are continuously being enhanced, there is still much room for improvement in the area of rheumatic diseases. As an example of this, the three tissues that are most affected in this kind of pathologies: cartilage, synovium and bone, have not yet been explored in the Human Protein Atlas initiative [47]. Therefore, our research group is developing an initiative within our hospital (DIPROA project) in order to join the proteomics data that have been acquired after more than ten years of investigation on joint tissues together with all the clinical data from the patients. All this information is being employed to build a business intelligence (BI) system combining data warehousing, online analytical processing (OLAP) and knowledge discovery by data mining (DM). This will be a highly valuable tool for knowledge management, allowing innovation in the health care practice that is currently being carried out in the Rheumatology Services.

\section{Conclusions}

The B/D platform of the Spanish HPP consortium is the core for the development of proteomic studies on cancer, obesity, cardiovascular, infectious and rheumatic diseases. The initiative on rheumatic diseases will apply its experience in the study of joint cells, tissues and body fluids for improving osteoarthritis research on the three pillars of the HPP project, which will be undoubtedly useful for addressing the important unmet clinical needs of this disease and other rheumatic pathologies.

\section{Transparency Document}

The Transparency document associated with this article can be found, in the online version.

\section{Conflict of interest disclosure}

The authors declare no competing financial interest.

\section{Acknowledgments}

This work was funded by grants from Fondo Investigación Sanitaria-Spain (PI11/02397, PI12/00329, CIBER-CB06/01/0040, RETIC-RIER-RD12/0009/0018) and Secretaría I + D + I Xunta de Galicia (10CSA916058PR). J. Mateos (CA11/00050) and P. Fernández-Puente (ProteoRed/ISCIII) are supported by Fondo Investigación Sanitaria-Spain. C. Ruiz-Romero is supported by the Miguel Servet program from Fondo Investigación Sanitaria-Spain (CP09/00114).

\section{R E F E R E N E S}

[1] Legrain P, Aebersold R, Archakov A, Bairoch A, Bala K, Beretta $\mathrm{L}$, et al. The human proteome project: current state and future direction. Mol Cell Proteomics 2011;10(7):M111 (009993).

[2] Paik YK, Jeong SK, Omenn GS, Uhlen M, Hanash S, Cho SY, et al. The Chromosome-Centric Human Proteome Project for cataloging proteins encoded in the genome. Nat Biotechnol 2012;30(3):221-3

[3] Pritzker K. Pathology of osteoarthritis. In: Brandt K, Doherty M, Lohmander L, editors. Osteoarthritis. New York: Oxford University Press; 1998. p. 50-61.

[4] Castro-Santos P, Laborde CM, Diaz-Pena R. Genomics, proteomics and metabolomics: their emerging roles in the discovery and validation of rheumatoid arthritis biomarkers. Clin Exp Rheumatol 2015 (Epub ahead of print).

[5] Ruiz-Romero C, Blanco FJ. Achievements and challenges of proteomics in the study of rheumatoid arthritis. Curr Top Med Chem 2013;13(6):732-42.

[6] Elding H, Lau W, Swallow DM, Maniatis N. Dissecting the genetics of complex inheritance: linkage disequilibrium mapping provides insight into Crohn disease. Am J Hum Genet 2011;89(6):798-805.

[7] Deng Y, Tsao BP. Genetic susceptibility to systemic lupus erythematosus in the genomic era. Nat Rev Rheumatol 2010; 6(12):683-92.

[8] Nath SK, Han S, Kim-Howard X, Kelly JA, Viswanathan P, Gilkeson GS, et al. A nonsynonymous functional variant in integrin-alpha(M) (encoded by ITGAM) is associated with systemic lupus erythematosus. Nat Genet 2008;40(2): $152-4$.

[9] Ruiz-Romero C, Blanco FJ. The role of proteomics in osteoarthritis pathogenesis research. Curr Drug Targets 2009;10(6):543-56.

[10] Ruiz-Romero C, Blanco FJ. Proteomics role in the search for improved diagnosis, prognosis and treatment of osteoarthritis. Osteoarthritis Cartilage 2010;18(4):500-9.

[11] Ruiz-Romero C, Carreira V, Rego I, Remeseiro S, Lopez-Armada MJ, Blanco FJ. Proteomic analysis of human osteoarthritic chondrocytes reveals protein changes in stress and glycolysis. Proteomics 2008;8(3):495-507.

[12] Ruiz-Romero C, Calamia V, Rocha B, Mateos J, Fernandez-Puente P, Blanco FJ. Hypoxia conditions differentially modulate human normal and osteoarthritic chondrocyte proteomes. J Proteome Res 2010;9(6):3035-45.

[13] Cillero-Pastor B, Ruiz-Romero C, Carames B, Lopez-Armada MJ, Blanco FJ. Proteomic analysis by two-dimensional electrophoresis to identify the normal human chondrocyte proteome stimulated by tumor necrosis factor alpha and interleukin-1beta. Arthritis Rheum 2010;62(3):802-14.

[14] Calamia V, Ruiz-Romero C, Rocha B, Fernandez-Puente P, Mateos J, Montell E, et al. Pharmacoproteomic study of the 
effects of chondroitin and glucosamine sulfate on human articular chondrocytes. Arthritis Res Ther 2010;12(4):R138.

[15] Ruiz-Romero C, Lopez-Armada MJ, Blanco FJ. Mitochondrial proteomic characterization of human normal articular chondrocytes. Osteoarthritis Cartilage 2006;14(6):507-18.

[16] Ruiz-Romero C, Calamia V, Mateos J, Carreira V, Martinez-Gomariz M, Fernandez M, et al. Mitochondrial dysregulation of osteoarthritic human articular chondrocytes analyzed by proteomics: a decrease in mitochondrial superoxide dismutase points to a redox imbalance. Mol Cell Proteomics 2009;8(1):172-89.

[17] Blanco FJ, Rego I, Ruiz-Romero C. The role of mitochondria in osteoarthritis. Nat Rev Rheumatol 2011;7(3):161-9.

[18] Rego I, Fernandez-Moreno M, Fernandez-Lopez C, Gomez-Reino JJ, Gonzalez A, Arenas J, et al. Role of European mitochondrial DNA haplogroups in the prevalence of hip osteoarthritis in Galicia, Northern Spain. Ann Rheum Dis 2010;69(1):210-3.

[19] Calamia V, Rocha B, Mateos J, Fernandez-Puente P, Ruiz-Romero C, Blanco FJ. Metabolic labeling of chondrocytes for the quantitative analysis of the interleukin-1-beta-mediated modulation of their intracellular and extracellular proteomes. J Proteome Res 2011;10(8):3701-11.

[20] Calamia V, Fernandez-Puente P, Mateos J, Lourido L, Rocha B, Montell E, et al. Pharmacoproteomic study of three different chondroitin sulfate compounds on intracellular and extracellular human chondrocyte proteomes. Mol Cell Proteomics 2012;11(6):M111 (013417).

[21] Calamia V, Lourido L, Fernández-Puente P, Mateos J, Rocha B, Montell E, et al. Secretome analysis of chondroitin sulfate-treated chondrocytes reveals anti-angiogenic, anti-inflammatory and anti-catabolic properties. Arthritis Res Ther 2012;14(5):R202.

[22] Guo D, Tan W, Wang F, Lv Z, Hu J, Lv T, et al. Proteomic analysis of human articular cartilage: identification of differentially expressed proteins in knee osteoarthritis. Joint Bone Spine 2008;75(4):439-44.

[23] Wu J, Liu W, Bemis A, Wang E, Qiu Y, Morris EA, et al. Comparative proteomic characterization of articular cartilage tissue from normal donors and patients with osteoarthritis. Arthritis Rheum 2007;56(11):3675-84.

[24] Zhen EY, Brittain IJ, Laska DA, Mitchell PG, Sumer EU, Karsdal MA, et al. Characterization of metalloprotease cleavage products of human articular cartilage. Arthritis Rheum 2008; 58(8):2420-31.

[25] Peffers MJ, Beynon RJ, Clegg PD. Absolute quantification of selected proteins in the human osteoarthritic secretome. Int J Mol Sci 2013;14(10):20658-81.

[26] Lourido L, Calamia V, Mateos J, Fernandez-Puente P, Fernandez-Tajes J, Blanco FJ, et al. Quantitative proteomic profiling of human articular cartilage degradation in osteoarthritis. J Proteome Res 2014;13(12):6096-106.

[27] Lotz M, Martel-Pelletier J, Christiansen C, Brandi ML, Bruyere O, Chapurlat R, et al. Value of biomarkers in osteoarthritis: current status and perspectives. Ann Rheum Dis 2013;72(11):1756-63.

[28] de Seny D, Sharif M, Fillet M, Cobraiville G, Meuwis MA, Maree $\mathrm{R}$, et al. Discovery and biochemical characterisation of four novel biomarkers for osteoarthritis. Ann Rheum Dis 2011; 70(6):1144-52.

[29] Takinami Y, Yoshimatsu S, Uchiumi T, Toyosaki-Maeda T, Morita A, Ishihara T, et al. Identification of potential prognostic markers for knee osteoarthritis by serum proteomic analysis. Biomark Insights 2013;8:85-95.

[30] Fernandez-Puente P, Mateos J, Fernandez-Costa C, Oreiro N, Fernandez-Lopez C, Ruiz-Romero C, et al. Identification of a panel of novel serum osteoarthritis biomarkers. J Proteome Res 2011;10(11):5095-101.

[31] Mateos J, Lourido L, Fernández-Puente P, Calamia V, Fernández-López C, Oreiro N, et al. Differential protein profiling of synovial fluid from rheumatoid arthritis and osteoarthritis patients using LC-MALDI TOF/TOF. J Proteomics 2012;75(10):2869-78.

[32] Han MY, Dai JJ, Zhang Y, Lin Q Jiang M, Xu XY, et al. Identification of osteoarthritis biomarkers by proteomic analysis of synovial fluid. J Int Med Res 2012;40(6):2243-50.

[33] Gobezie R, Kho A, Krastins B, Sarracino DA, Thornhill TS, Chase $\mathrm{M}$, et al. High abundance synovial fluid proteome: distinct profiles in health and osteoarthritis. Arthritis Res Ther 2007;9(2):R36.

[34] Ritter SY, Subbaiah R, Bebek G, Crish J, Scanzello CR, Krastins $B$, et al. Proteomic analysis of synovial fluid from the osteoarthritic knee: comparison with transcriptome analyses of joint tissues. Arthritis Rheum 2013;65(4):981-92.

[35] Verma P, Dalal K. Serum cartilage oligomeric matrix protein (COMP) in knee osteoarthritis: a novel diagnostic and prognostic biomarker. J Orthop Res 2013;31(7):999-1006.

[36] Zivanovic S, Rackov LP, Vojvodic D, Vucetic D. Human cartilage glycoprotein 39-biomarker of joint damage in knee osteoarthritis. Int Orthop 2009;33(4):1165-70.

[37] Hsueh MF, Onnerfjord P, Kraus VB. Biomarkers and proteomic analysis of osteoarthritis. Matrix Biol 2014;39c:56-66.

[38] Bauer DC, Hunter DJ, Abramson SB, Attur M, Corr M, Felson D, et al. Classification of osteoarthritis biomarkers: a proposed approach. Osteoarthritis Cartilage 2006;14(8):723-7.

[39] Kraus VB, Burnett B, Coindreau J, Cottrell S, Eyre D, Gendreau M, et al. Application of biomarkers in the development of drugs intended for the treatment of osteoarthritis. Osteoarthritis Cartilage 2011;19(5):515-42.

[40] Aebersold R, Bader GD, Edwards AM, van Eyk JE, Kussmann $\mathrm{M}$, Qin J, et al. The biology/disease-driven human proteome project (B/D-HPP): enabling protein research for the life sciences community. J Proteome Res 2013;12(1):23-7.

[41] Surinova S, Huttenhain R, Chang CY, Espona L, Vitek O, Aebersold R. Automated selected reaction monitoring data analysis workflow for large-scale targeted proteomic studies. Nat Protoc 2013;8(8):1602-19.

[42] Liu Y, Huttenhain R, Collins B, Aebersold R. Mass spectrometric protein maps for biomarker discovery and clinical research. Expert Rev Mol Diagn 2013;13(8):811-25.

[43] Ritter SY, Collins J, Krastins B, Sarracino D, Lopez M, Losina E, et al. Mass spectrometry assays of plasma biomarkers to predict radiographic progression of knee osteoarthritis. Arthritis Res Ther 2014;16(5):456.

[44] Fagerberg L, Oksvold P, Skogs M, Algenas C, Lundberg E, Ponten F, et al. Contribution of antibody-based protein profiling to the human Chromosome-centric Proteome Project (C-HPP). J Proteome Res 2013;12(6):2439-48.

[45] Chandra PE, Sokolove J, Hipp BG, Lindstrom TM, Elder JT, Reveille JD, et al. Novel multiplex technology for diagnostic characterization of rheumatoid arthritis. Arthritis Res Ther 2011;13(3):R102.

[46] Henjes F, Lourido L, Ruiz-Romero C, Fernandez-Tajes J, Schwenk JM, Gonzalez-Gonzalez M, et al. Analysis of autoantibody profiles in osteoarthritis using comprehensive protein array concepts. J Proteome Res 2014;13(11):5218-29.

[47] Uhlen M, Oksvold P, Fagerberg L, Lundberg E, Jonasson K, Forsberg $\mathrm{M}$, et al. Towards a knowledge-based human protein atlas. Nat Biotechnol 2010;28(12):1248-50. 